\title{
Le SEO pour votre cabinet d'optométrie : la « formule secrète » toujours efficace en 2020
}

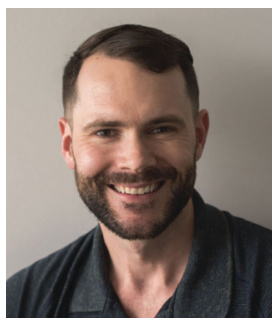

Par : Cameron Martel, gestionnaire - SEO et solutions de contenu @ 4ECPs Cameron Martel est un spécialiste du marketing numérique chevronné qui gère des campagnes de SEO et de contenu depuis 2005. Il travaille actuellement avec des dizaines de cabinets de soins oculovisuels dans le cadre de son travail avec Marketing4ECPs. Il est daltonien, mais ne le lui rappelez pas, sinon il verra rouge! (ou du moins, c'est ce qu'il croit). Pour joindre Cameron : www.marketing4ecps.com ou cameron@4ecps.com.

$\mathrm{J}$ e vais partager avec vous un petit secret à propos du référencement naturel, aussi appelé optimisation pour les moteurs de recherche (search engine optimization - SEO), que la plupart des professionnels de ce domaine ne veulent pas que vous sachiez : pour la plupart des optométristes, le SEO n'est pas difficile... et ça n'a pas besoin d'être compliqué non plus. Cependant, comme des « spécialistes du SEO » malintentionnés tentent depuis des années de les persuader du contraire, les propriétaires d'entreprise ont l'impression que le SEO est tout sauf simple.

Êtes-vous de cet avis? Si c'est le cas, nous voulons modifier cette perception dès aujourd'hui.

CE QUE LE SEO N'EST PAS

Les professionnels du SEO «à la morale douteuse » existent depuis aussi longtemps que les moteurs de recherche eux-mêmes, et ils sont reconnus pour faire des promesses qu'ils ne semblent jamais tenir. Il n'est donc pas étonnant que, pour de nombreux optométristes, le SEO équivaut à une sorte de boîte noire mystérieuse.

Sachant cela, avant d'aborder en quoi consiste le SEO, précisons ce qu'il n'est pas :

- Mystérieux - Le SEO devrait être un processus transparent comprenant des éléments de flux des travaux et des échéanciers clairs.

- Coûteux - L’investissement dans le SEO s'harmonise généralement avec d'autres types de marketing axé sur le rendement. Si la soumission est élevée, demandez à voir la ventilation du budget.

- Cloisonné - Le SEO ne devrait jamais exister par lui-même comme votre unique méthode de marketing. Il devrait toujours être complété grâce aux médias traditionnels et à des tactiques de marketing axé sur le rendement.

- Rapide - Il peut s'écouler quelques semaines avant que les efforts de SEO ne portent leurs fruits (Google peut mettre du temps à déterminer où se situe systématiquement un site Web dans son index).

LE SEO, QU'EST-CE QUE C'EST?

Maintenant que vous savez ce que le SEO n'est pas, décrivons ce qu'il est :

- Transparent - Le travail de SEO n'est pas intangible; c'est un mélange de stratégie de blogue, de création de contenu, de gestion de la réputation et de supervision technique de site Web.

- Abordable - Le SEO n'est pas nécessairement coûteux, à moins que vous prévoyiez un référencement à l'échelle nationale ou internationale.

- Tourné vers l'utilisateur - Plus vous ajoutez de valeur à l'équation (du point de vue de l'utilisateur), plus votre SEO sera solide. 
- Mesurable - Vous pouvez mesurer des éléments, comme le trafic généré par les résultats naturels (dans Google Analytics), pour voir l'impact de vos efforts de SEO au fil du temps. Pensez en trimestres plutôt qu'en jours ou en semaines.

LA FORMULE SECRÈTE POUR LAQUELLE LES OPTOMÉTRISTES PAIENT TROP CHER

Que vous gériez votre SEO vous-même ou que vous souhaitiez mieux comprendre et évaluer votre équipe de SEO, c'est une bonne idée d'élaborer une stratégie qui reprend, dans l'ordre ou le désordre, les points suivants. J'ai énuméré ces points dans l'ordre d'importance que je préfère :

1. Assurez-vous de revendiquer et d'optimiser votre profil Google Mon Entreprise - Le revendiquer n'est pas suffisant. Optimisez-le :

a. en ajoutant des photos de l'intérieur/l'extérieur de votre cabinet, des membres de votre équipe, de votre logo, etc.

b. en vous assurant que le nom, l'adresse et le numéro de téléphone correspondent à ce qui est indiqué sur votre site Web, ainsi qu'aux principaux répertoires et profils (sites comme pagesjaunes.ca, Yelp!, LinkedIn, etc.).

c. en répondant aux avis, qu'ils soient positifs ou négatifs.

2. Établissez les caractéristiques fondamentales de votre site Web - Veillez à ce que votre site Web respecte les pratiques exemplaires techniques qui sont largement publiées sur le Web (Moz a un bon guide à ce sujet). Quelques détails qui comptent vraiment dans le SEO actuellement :

a. Votre site Web devrait avoir une page dédiée pour chaque service médical, p. ex., la sécheresse oculaire.

b. Assurez-vous que votre site Web se charge rapidement (moins de trois secondes sur un ordinateur de bureau, deux secondes et demie sur un appareil mobile).

c. Faites en sorte qu'il soit facile d'accéder à n'importe quelle page ou publication sur votre site en trois clics ou moins.

3. Faites de votre page « À propos de nous » une expérience agréable - C'est important, non seulement parce que votre page « À propos de nous » est susceptible d'être une des dix pages les plus consultées de votre site Web, mais aussi parce que c'est là que vous démontrez votre savoir-faire, ce que Google privilégie de plus en plus.

4. Établissez vos profils de médias sociaux et utilisez-les - Je vous recommande de vous concentrer sur Facebook et YouTube, car ce sont les plateformes les plus conviviales pour mobiliser votre public. Soyez efficace et créez du contenu qui favorise la participation et les discussions.

5. Créez une expérience de blogue qui vaut la peine d'être lue (offrez un contenu complet et de qualité) - Plus le contenu que vous offrez est exceptionnel, plus Google vous rendra visible, et plus cela génèrera de trafic pour vous.

6. Partagez votre contenu avec vos pairs de l'industrie, les sites Web pertinents et les médias sociaux locaux - Présentez votre contenu à votre public et, mieux encore, incitez-le à en parler!

VOILÀ; VOUS CONNAISSEZ MAINTENANT LA FORMULE SECRÈTE!

Simple, n'est-ce pas? Une fois les caractéristiques de votre site Web bien établies, votre stratégie SEO change de cap pour se concentrer sur la création de contenu exceptionnel et amener votre communauté à parler de vous.

Voilà ce que sera le SEO en 2020, et probablement aussi en 2021. • 